\title{
THE DIFFERENCES IN RESPONSE TO ACUTE STRESS OF INDIVIDUALS WITH HIGHER AND LOWER NEUROTICISM
}

\author{
Gabija Jarašiūnaitė-Fedosejeva, Erika Varnagirytė, \& Aidas Perminas \\ Department of Psychology, Vytautas Magnus University (Lithuania)
}

\begin{abstract}
Although some studies analyze neuroticism's role in individuals' response to acute stress, the results are controversial. There is a lack of studies examining the response to stressors of individuals with higher and lower neuroticism in all phases (during the period of anticipation of the stressor, at the time of exposure to the stressor, and during the recovery period after exposure to a stressor), measuring different physiological parameters and evaluating emotional response to a stressor at the same time. This study aimed to assess individuals with higher and lower neuroticism physiological and emotional responses to acute stress. 168 students participated in a study ( 23 males and 145 females). Their response to 4 different stressors (1 physical and 3 psychological (with standard instruction, the pressure to compete and critique) was evaluated, measuring the changes in their skin conductance, skin temperature, heart rate, respiratory rate while waiting for the stressor (anticipation phase), during the stressor and in the stress recovery phase. The changes in students 'emotional responses were also measured using the C.R. Carlson et al. (1989) Emotional Assessment Scale (EAS). Students' neuroticism was assessed using the NEO Five-Factor Inventory's neuroticism subscale (NEO-FFI, Costa, McCrae, 1992). The study results showed that students having higher and lower neuroticism differed when reacting to a physiological stressor. Students' responses to a psychological stressor differed only in the condition when they were criticized.
\end{abstract}

Keywords: Neuroticism, physiological response to stress, emotional response to stress, acute stress.

\section{Introduction}

The relationship between personality traits and stress response has been studied for several decades. Studies show that different personalities' responses to acute stress may vary (Ellis, Jackson \& Boyce, 2006). This is determined by numerous factors, from genetics to environment (Hutchinson \& Ruiz, 2011). Among various personality classifications, the most recently researched is the Big Five trait taxonomy, which consists of five personality dimensions: extraversion, neuroticism, conscientiousness, agreeableness, and openness (Bibbey, Carroll, Roseboom, Phillips \& de Rooij, 2013).

Longitudinal research reveals that neuroticism, which is considered as person's tendency to experience negative feelings like fear, sadness, dissatisfaction, anger, and guilt (McCrae \& Costa, 1987), is not only related to poorer young adult's current well-being and life satisfaction but also have a lasting effect on well-being several decades later. Authors also highlight that this impact is mostly indirect and is exposed by a person's vulnerability to stress (Gale, Booth, Mõttus, Kuh \& Deary, 2013). Aside, neuroticism is related to plenty of negative physical and psychological health consequences (Ormel et al., 2013).

Stress-moderation models indicate that personality can impact the biological stress response system (Hutchinson \& Ruiz, 2011). Numerous studies highlight that neuroticism has the strongest relation to stress than other Big Five traits (Coyle, Howard, Bibbey, Gallagher, Whittaker \& Creaven, 2020). Even the research results of the relationship between acute stress and neuroticism are controversial, yet mostly confirmed is this trait relation to higher emotional stress reactivity (Suls \& Martin, 2005), but lower cortisol and cardiovascular stress reactivity (Bibbey et al., 2013; Coyle et al., 2020).

According to Bibbey et al. (2013), individuals' responses to acute stress may also vary depending on the stressor type. The study results showed that lower cortisol, heart rate, systolic and diastolic blood pressure were found in individuals with higher neuroticism when reacting to psychological stressors. Coyle et al. (2020) replicated the described study and found that neuroticism was negatively related to cortisol reactivity. Moreover, Poppelaars, Klackl, Pletzer, Wilhelm, and Jonas (2019) found that higher neuroticism predicted lower sympathetic nervous system reactivity at the time of exposure to a 
psychological stressor. During the recovery period, cortisol recovered more slowly for those higher in neuroticism. However, no differences were found during the anticipation phase of the stressor.

The study by Hutchinson and Ruiz (2011) has been done only in the women sample. The authors explored the effects of neuroticism on psychophysiological response to an interpersonal stressor. The results showed no significant relation between neuroticism and cardiovascular and emotional reactivity to stressor or recovery after the stressor. The blood pressure recovery was poorer for these women, who were characterized as having higher neuroticism. For comparison, Jonassaint et al. (2009) provided two psychological tasks to male police officers: mental arithmetic and anger recall. Results revealed that males higher in neuroticism responded with lower diastolic blood pressure.

As discussed, there is a lack of studies examining the response to stressors of individuals with higher and lower neuroticism in all stress response phases (when waiting for the stressor, at the time of exposure to the stressor, and during the recovery period after exposure to a stressor) while measuring different physiological parameters and evaluating emotional response. Additionally, existing studies assessed response only to psychological stressors; no studies analyze the response to a physiological stressor or explore them together at the same research. Consequently, this study aimed to assess individuals with higher and lower neuroticism physiological and emotional responses to acute stress.

\section{Methods}

Study sample. 168 students aged between 18 and 31 participated in a study (23 males and 145 females). Their scores on the neuroticism scale varied from 28.0 to 84.4 (mean 54.2 , st. deviation 11.31). Students were assigned as having lower $(\mathrm{N}=84)$ or higher neuroticism $(\mathrm{N}=84)$ based on the mean on the scale. All participants were healthy, with no chronic non-communicable diseases, free of cardioactive medicines, sedatives, and antidepressants. There were no significant differences in any relevant background variables (such as depressive symptoms, phase of the menstrual cycle (for females), number of exams per week or day in a study period) among higher and lower students' neuroticism.

Measures. Students' neuroticism was evaluated using the NEO Five-Factor Inventory's neuroticism subscale (NEO-FFI, Costa, McCrae, 1992). The subscale has demonstrated a good internal consistency. For the current sample, Cronbach's $\alpha$ was 0.83 . Students filled this questionnaire after all stressor tasks.

Students' emotional reactivity to stressors was measured using the Emotional Assessment Scale (EAS, Carlson et al., 1989). The EAS comprises 24 adjectives divided into eight emotion categories, but only five of them (anger, anxiety, fear, sadness, and guilt) were analyzed in this study. Participants of the study were asked to fill the scale before the stressor tasks and after each task on how they felt during the tasks. The internal consistency between subscales varied from 0.513 to 0.884 .

Physiological response to stressors was assessed using MindMedia device NeXus - 10 with Biotrace+ software. Physiological response to stressors was assessed by measuring reactivity during the anticipation and exposure to stressors, and recovery. The physiological response was assessed by skin conductance, skin temperature, heart rate, and respiratory rate. Skin conductance was measured in micro-ohms, while skin temperature was measured on the scale of Celsius. The heart rate was measured by the number of heartbeats per minute, while the respiratory rate by breath cycles per minute.

A total of 4 stressors ( 3 psychological and 1 physiological) was used to assess students' response to stressors. Bourdon test, which measures attention concentration, was used as a psychological stressor. During the psychological stressor task, the participants were given a printed-paper with a 693-letter matrix and were asked to cross out the letters "a," "b," "q" as soon as possible and without mistakes. The participants were asked to perform three equal tasks while given different situations: when giving basic instructions, when reminding time restrictions, and providing stimulus to compete and when criticizing while doing the task. A cold pressor test was used as a physiological stressor. During it, the participants were asked to keep their dominant hand in cold water $\left(4^{\circ} \mathrm{C}\right)$ for 1 minute.

Procedure. All students were tested individually in the psychology laboratory, consisting of one middle-sized room (temperature $23-24^{\circ} \mathrm{C}$ ). Students were asked to abstain from alcohol, other drugs, and strenuous exercise for 12 hours and caffeine and smoking for 4 hours before the quasi-experiment. Upon arrival to the laboratory, participants signed an informed consent form that described the study's procedures, benefits and potential harm, confidentiality, and participants' rights. Then they were asked to fill out a packet of questionnaires about their health condition, emotions before an experiment, and some sociodemographic variables. After that, the sensors measuring physiological response to stressor (skin conductance, skin temperature, heart rate) were placed on students' non-dominant hand fingers and waist (sensor measuring respiratory rate). Instead of a usual "vanilla" baseline task, students were asked to lie on a couch and try to relax for 10 minutes and were told that we measure their ability to relax. 
During the first psychological task (stressor), the experimenter gave a brief general instruction, that when given a sign, a student would be asked to cross out the letters "a," "b," "q," and he/she needs to do it as fast as he/she could and try to make no errors. The experimenter mentions that the student also would be given a sign when to finish the task. When instructing a second task (stressor), an experimenter told the participant that most of the students perform the task in $1.5 \mathrm{~min}$., but he/she would be given 2 minutes. When given a sign, a student would need to cross out the letters "a," "b," "q," and he/she needs to do it as fast as he/she could and try to make no errors. The experimenter also mentions that the student would be told when $1 \mathrm{~min}$. and $30 \mathrm{~s}$. would be left for the performance of the task. Such instructions were created to arouse a desire to compete. The instructions for the third task (stressor) sounded the same as for the first. Just when a student began to perform a task after every $15 \mathrm{~s}$. of performance, he would be told a critique; "Your score would depend on how fast you will do the task," "Can't you do it faster?" "Probably today you are very tired," "I think that you are not doing very well," "Are you trying hard enough?". That critique was said to provoke hostility. During the fourth stressor, the participant was presented with a bowl of cold water and, when given a sign, was asked to put /her hand in the water and keep it for 1 minute.

Students' physiological responses to stressors were recorded at four phases: baseline (2.5 min.), instruction announcement $(0.5 \mathrm{~min}$.), task ( $2 \mathrm{~min}$. for psychological stressors and $1 \mathrm{~min}$. for physiological stressor), and recovery period ( $2 \mathrm{~min}$.). After performing each task, the subjects were asked to complete the EAS based on their feelings during the task. The time interval between each stressor task was around 2 min.

Based on methodological recommendations for comparing stress response (Burt, Obradovic, 2013; Smith, Unchino, 2007), change scores of physiological variables from the initial value were analyzed instead of raw scores. Change scores were calculated by subtracting baseline values from physiological indicators' values in anticipation, exposure to the stressor, and recovery phases. In this case, higher positive skin conductance, heart rate, and respiratory rate change score in anticipation and exposure to stressor phases shows greater skin conductance, heart rate, and respiratory rate reactivity, while a higher negative change score shows greater skin temperature reactivity. When higher positive skin conductance, heart rate, and respiratory rate change scores in the recovery phase show slower recovery after the stressor, a smaller positive or negative change score measured by the same variables shows faster recovery after stressors. Contrarily, a higher negative skin temperature change score shows slower recovery after the stressor, while a smaller negative or positive score shows faster skin temperature recovery.

The emotional response was evaluated by change scores in five emotions: anger, anxiety, fear, sadness, and guilt. Change scores were calculated by subtracting baseline values of particular emotion from that emotion felt during the stressor phase. In this case, higher positive change scores of measured emotions show greater emotional reactivity. Higher negative change scores show that subjects had higher scores of that emotion in the baseline than during a particular stressor.

Data analysis. Shapiro-Wilk test showed that physiological response changes had a normal distribution, so analysis of covariance was used when analyzing students' physiological response to stressors. An independent sample t-test was used to examine the baseline equivalency of physiological variables. However, the data of emotional response changes to stressors deviated from a normal distribution, so the Mann-Whitney $U$ test was used to compare the emotional response to stressors between different study groups. The statistical significance level of data analysis was 0.05 .

\section{Results}

Independent sample t-test showed no significant differences between students with higher and lower neuroticism at baseline for any of the four physiological indices. However, students with lower neuroticism had statistically significantly higher anger, anxiety, fear, guilt, and sadness at the baseline, before starting the stressor tasks $(\mathrm{p}<0.05)$.

The comparison of physiological response to stressors of individuals with higher and lower neuroticism is presented in Table 1. Only results representing statistical differences are reported in a table. The study results showed no differences between students with higher and lower neuroticism physiological responses to the first two psychological stressors. Also, there were no statistically significant differences in heart rate and respiratory rate response between students with higher and lower neuroticism when facing third and fourth stressors. However, students with higher neuroticism had greater skin temperature reactivity $\left(\mathrm{F}(1)=3.538 ; \eta^{2}=0.023 ; \mathrm{p}<0,05\right)$ to the third stressor when they were criticized and slower skin temperature recovery $\left(F(1)=5.278 ; \eta^{2}=0.034 ; p<0,05\right)$ after stressor than individuals with lower neuroticism. Though, students with higher neuroticism had lower skin conductance reactivity in the anticipation phase $\left(\mathrm{F}(1)=4.183, \eta^{2}=0.026, \mathrm{p}<0.05\right)$ while hearing the instructions and waiting for the stressor than students with lower neuroticism. 
Table 1. Comparison of physiological response to stressors of individuals with higher and lower neuroticism.

\begin{tabular}{|c|c|c|c|c|c|c|c|}
\hline \multirow{3}{*}{ PR } & \multirow{3}{*}{$\begin{array}{c}\text { Stressor } \\
\text { phase }\end{array}$} & \multicolumn{3}{|c|}{ 3rd stressor } & \multicolumn{3}{|c|}{ 4th stressor } \\
\hline & & \multicolumn{2}{|c|}{ Mean $( \pm$ SD) } & \multirow[b]{2}{*}{$\mathbf{p}$} & \multicolumn{2}{|c|}{ Mean ( \pm SD) } & \multirow[b]{2}{*}{$\mathbf{p}$} \\
\hline & & $\begin{array}{c}\text { Higher } \\
\text { neuroticism }\end{array}$ & $\begin{array}{c}\text { Lower } \\
\text { neuroticism }\end{array}$ & & $\begin{array}{c}\text { Higher } \\
\text { neuroticism }\end{array}$ & $\begin{array}{c}\text { Lower } \\
\text { neuroticism }\end{array}$ & \\
\hline \multirow{3}{*}{$\mathrm{SC}$} & Anticipation & $0.168(0.262)$ & $0.147(0.346)$ & 0.624 & $0.204(0.297)$ & $0.301(0.340)$ & 0.043 \\
\hline & Stressor & $0.475(0.448)$ & $0.580(0.516)$ & 0.189 & $0.191(0.333)$ & $0.327(0.529)$ & 0.053 \\
\hline & Recovery & $0.167(0.259)$ & $0.199(0.292)$ & 0.440 & $-0.066(0.197)$ & $-0.037(0.286)$ & 0.421 \\
\hline \multirow{3}{*}{ TMP } & Anticipation & $0.183(0.446)$ & $0.309(0.491)$ & 0.097 & $0.094(0.464)$ & $0.039(0.408)$ & 0.442 \\
\hline & Stressor & $-0.242(0.593)$ & $-0.053(0.670)$ & 0.049 & $-0.093(0.572)$ & $-0.174(0.558)$ & 0.288 \\
\hline & Recovery & $-0.417(0.852)$ & $-0.111(0.831)$ & 0.023 & $-0.414(0.713)$ & $-0.442(0.674)$ & 0.639 \\
\hline
\end{tabular}

$* P R-$ physiological reactivity, $S C-$ skin conductance, TMP - skin temperature.

The comparison of emotional response to stressors of individuals with higher and lower neuroticism is presented in Table 2. The results indicated that individuals with higher neuroticism had a lower emotional response (measured by anger, anxiety, guilt, and sadness) to the psychological stressor when they were criticized than students with lower neuroticism $(p<0.005)$. Both groups (students with higher and lower neuroticism) had negative guilt change scores, which means that they felt less guilty during the stressor than in the baseline (before stressor tasks). However, the response of students with higher neuroticism was less negative, which means that they felt more guilt, than students with lower neuroticism $(\mathrm{p}<0.05)$.

Table 2. Comparison of emotional response to stressors of individuals with higher and lower neuroticism.

\begin{tabular}{|c|c|c|c|c|c|c|}
\hline \multirow{3}{*}{$\begin{array}{l}\text { Emotional } \\
\text { reactivity }\end{array}$} & \multicolumn{3}{|c|}{ 3rd stressor } & \multicolumn{3}{|c|}{ 4th stressor } \\
\hline & \multicolumn{2}{|c|}{ Mean $( \pm$ SD) } & \multirow[b]{2}{*}{$\mathbf{p}$} & \multicolumn{2}{|c|}{ Mean $( \pm$ SD) } & \multirow[b]{2}{*}{$\mathbf{p}$} \\
\hline & $\begin{array}{c}\text { Higher } \\
\text { neuroticism }\end{array}$ & $\begin{array}{c}\text { Lower } \\
\text { neuroticism }\end{array}$ & & $\begin{array}{c}\text { Higher } \\
\text { neuroticism }\end{array}$ & $\begin{array}{c}\text { Lower } \\
\text { neuroticism }\end{array}$ & \\
\hline Anger & $4.702(11.547)$ & $11.028(19.692)$ & 0.028 & $1.552(9.099)$ & $1.943(13.623)$ & 0.569 \\
\hline Anxiety & $-1.518(18.468)$ & $4.964(23.145)$ & 0.045 & $-2.948(14.471)$ & $0.337(23.885)$ & 0.509 \\
\hline Fear & $-1.333(8.402)$ & $0.193(13.130)$ & 0.815 & $-0.516(9.117)$ & $3.449(16.594)$ & 0.144 \\
\hline Guilt & $0.758(6.364)$ & $5.144(11.512)$ & 0.036 & $-0.482(3.492)$ & $-2.815(7.581)$ & 0.014 \\
\hline Sadness & $1.076(11.076)$ & $5.539(16.609)$ & 0.041 & $0.748(9.141)$ & $2.498(13.965)$ & 0.631 \\
\hline
\end{tabular}

\section{Discussion}

The study showed that subjects with higher neuroticism had a higher physiological response (measured by skin temperature) to the psychological stressor where cognitive evaluation is required but had a lower physiological response (measured by skin conductance) to physiological stressor where the response is automatic. Surprisingly, students with higher neuroticism had a lower emotional response to the psychological stressor than subjects with lower neuroticism.

These findings partly contrast with earlier reported associations between response to acute stress and neuroticism: it was discussed that individuals with higher neuroticism usually respond with higher emotional but lower physiological stress reactivity (Bibbey et al., 2013; Coyle et al., 2020; Jonassaint et al. 2009). Poppelaars et al. (2019) determined that higher neuroticism predicts lower physiological response to a psychological stressor, but the opposite relationship was observed in this study. However, this study supplements Poppelaars et al. (2019) and Hutchinson and Ruiz (2011), that after stress, physiological characteristics recover more slowly for individuals with higher neuroticism.

The result that individuals with higher neuroticism have more neutral emotional but higher physiological responses to the psychological stressor is quite surprising. According to Evans et al. (2016), higher neuroticism is related to higher emotional responses when exposed to psychological stressors. Researchers point out that unequal emotional and physiological responses may also be associated with other individual differences (Childs, White, de Wit, 2014).

It is hypothesized that individuals higher in neuroticism may deny their emotional response and be afraid to appear more negative than they feel: negative fear and anxiety change scores partly support this prediction. Lee-Baggley, Preece and DeLongis (2005) adds that when reacting to stress, individuals having higher neuroticism tend to respond using passive and non-adaptive mechanisms like denial, avoidance, or self-blame.

Earlier studies have not evaluated physiological and emotional responses to psychological stressors with different instructions (e.g., in the situations that individuals need to rush and compete or are criticized). As this study showed, individuals with higher neuroticism might have different responses in 
different contexts, so it is important to modify psychological laboratory stressors to potential real-life situations and evaluate their response.

This study has some limitations. The first one is the quasi-experimental design of the study. The respondents were not randomly assigned to the group, but the assignment was based on personality characteristics. Secondly, we did not select our respondents based on their scores (highest and lowest) in the neuroticism subscale from a large sample of students. Another limitation is that we used dichotomized trait variables and compared the response to the stressor of individuals with lower and higher neuroticism. The results of the study can also be affected by unequal gender distribution through the sample groups. Besides, any of the health difficulties and used medicines weren't accessed objectively; we relied on the subjects' information.

\section{References}

Bibbey, A., Carroll, D., Roseboom, T. J., Phillips, A. C., \& de Rooij, S. R. (2013). Personality and physiological reactions to acute psychological stress. International Journal of Psychophysiology, 90(1), 28-36.

Burt, K. B., Obradovic, J. (2013) The Construct of Psychophysiological Reactivity: Statistical and Psychometric Issues, Developmental Review, 33(1), 29-57.

Carlson, C.R., Collins, F.L., Stewart, J.F., Porzelius, J., Nitz, J.A., Lind, C.O. (1989) The Assessment of Emotional Reactivity: A Scale Development and Validation Study, Journal of Psychology and Behavioral Assessment, 11(4), 313-325.

Childs, E., White, T. L., \& de Wit, H. (2014). Personality traits modulate emotional and physiological responses to stress. Behavioural Pharmacology, 25(5 0 6), 493-502.

Costa, P. T., \& McCrae, R. R. (1992). Revised Neo Personality Inventory (NEO-PI-R) and NEO Five-Factor Inventory (NEO-FFI). Psychological Assesment Resources.

Coyle, D. K., Howard, S., Bibbey, A., Gallagher, S., Whittaker, A. C., \& Creaven, A. M. (2020). Personality, cardiovascular, and cortisol reactions to acute psychological stress in the Midlife in the United States (MIDUS) study. International Journal of Psychophysiology, 148, 67-74.

Čukić, I., \& Bates, T. C. (2015). The association between neuroticism and heart rate variability is not fully explained by cardiovascular disease and depression. PloS one, 10(5).

Ellis, B. J., Jackson, J. J., \& Boyce, W. T. (2006). The stress response systems: Universality and adaptive individual differences. Developmental Review, 26(2), 175-212.

Evans, B. E., Stam, J., Huizink, A. C., Willemen, A. M., Westenberg, P. M., Branje, S., ... \& van Lier, P. A. (2016). Neuroticism and extraversion in relation to physiological stress reactivity during adolescence. Biological Psychology, 117, 67-79.

Gale, C. R., Booth, T., Mõttus, R., Kuh, D., \& Deary, I. J. (2013). Neuroticism and Extraversion in youth predict mental wellbeing and life satisfaction 40 years later. Journal of Research in Personality, 47(6), 687-697.

Hughes, B. M., Howard, S., James, J. E., \& Higgins, N. M. (2011). Individual differences in adaptation of cardiovascular responses to stress. Biological Psychology, 86(2), 129-136.

Hutchinson, J. G., \& Ruiz, J. M. (2011). Neuroticism and cardiovascular response in women: Evidence of effects on blood pressure recovery. Journal of Personality, 79(2), 277-302.

Jansson, B., \& Najström, M. (2009). Is preattentive bias predictive of autonomic reactivity in response to a stressor? Journal of Anxiety Disorders, 23(3), 374-380.

Jonassaint, C. R., Why, Y. P., Bishop, G. D., Tong, E. M., Diong, S. M., Enkelmann, H. C., ... \& Ang, J. (2009). The effects of neuroticism and extraversion on cardiovascular reactivity during a mental and an emotional stress task. International Journal of Psychophysiology, 74(3), 274-279.

Lee-Baggley, D., Preece, M., \& DeLongis, A. (2005). Coping with interpersonal stress: Role of Big Five traits. Journal of Personality, 73(5), 1141-1180.

McCrae, R. R., \& Costa, P. T. (1987). Validation of the five-factor model of personality across instruments and observers. Journal of Personality and Social Psychology, 52(1), 81-90.

Ormel, J., Bastiaansen, A., Riese, H., Bos, E. H., Servaas, M., Ellenbogen, M., ... \& Aleman, A. (2013). The biological and psychological basis of neuroticism: Current status and future directions. Neuroscience \& Biobehavioral Reviews, 37(1), 59-72.

Poppelaars, E. S., Klackl, J., Pletzer, B., Wilhelm, F. H., \& Jonas, E. (2019). Social-evaluative threat: Stress response stages and influences of biological sex and neuroticism. Psychoneuroendocrinology, 109, 104378.

Smith, T.W., Uchino, B.N. (2007) Measuring Physiological Processes in Biopsychosocial Research. In Luecken, L.J., Gallo, L.C. (eds.) Handbook of Physiological Research Methods in Health Psychology, Sage Publications, $472 \mathrm{p}$.

Suls, J., \& Martin, R. (2005). The daily life of the garden-variety neurotic: Reactivity, stressor exposure, mood spillover, and maladaptive coping. Journal of Personality, 73(6), 1485-1510. 